\title{
Raíces y tubérculos tropicales olvidados o subutilizados en Brasil
}

\section{Tropical root and tuber crops forgotten or underexploited in Brazil}

MARCOS VINÍCIUS BOHRER MONTEIRO SIOUEIRA'

ELIZABETH ANN VEASEY'
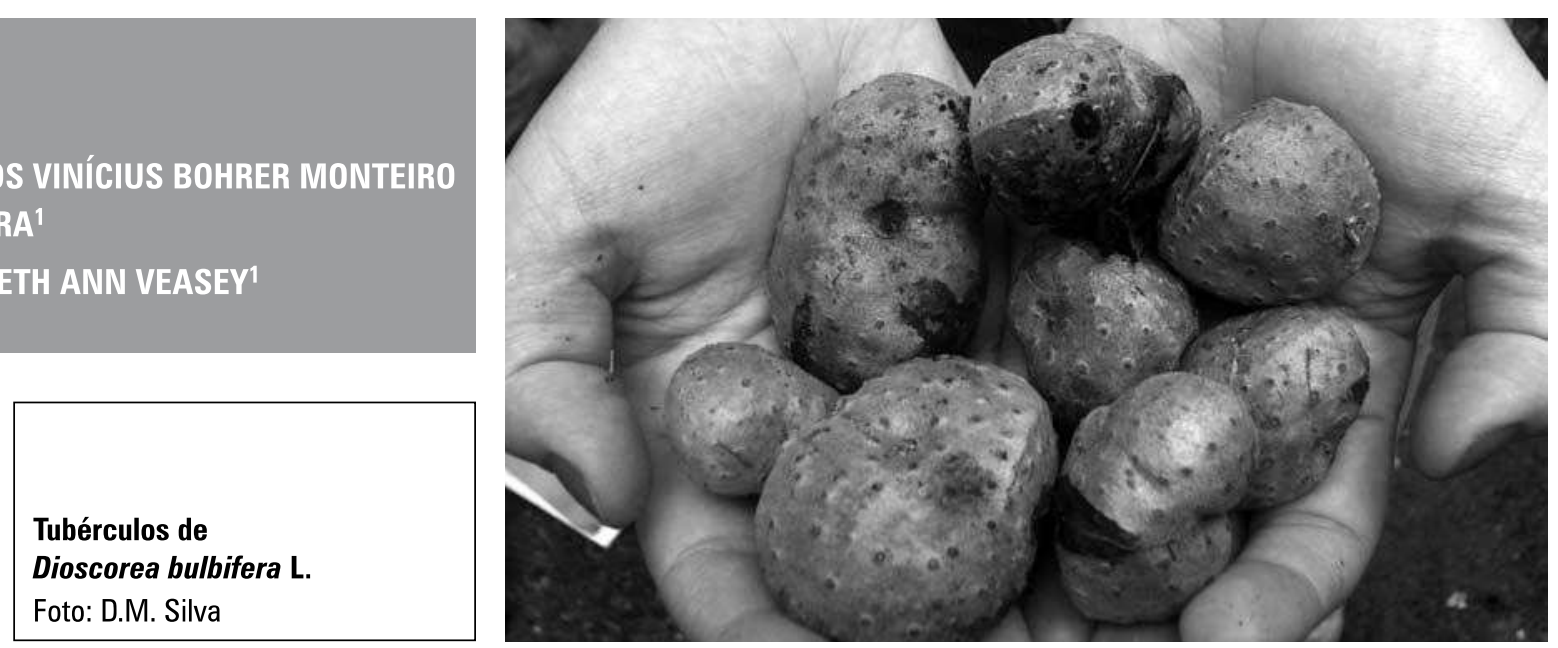

\section{RESUMEN}

La agricultura tradicional en Brasil básicamente es mantenida para fines de subsistencia en comunidades locales, sin el uso de tecnologías modernas, usufructuando la mano de obra familiar. Una amplia gama de especies son usadas por esos agricultores que mantienen gran diversidad de variedades de cada especie, sea dentro o entre quintales y chacras, presentando también un intenso sistema de intercambio de esos genotipos entre familiares y vecinos. De esa gran diversidad de cultivos, se destacan muchas tuberosas que son parte de la dieta de la mayoría de los brasileros, siendo la yuca, los ñames y la papa dulce (camote) las más cultivadas. A pesar de toda la importancia cultural y alimentaria, esas tuberosas demarcan un "olvido" por parte de grupos de investigación, extensionistas y sociedad en general, visto que son escasas las investigaciones asociadas a ellas, si comparamos con culturas de ciclo corto, en las que predominan la fuerza del agro negocio y el apoyo gubernamental. Cultivos "huérfanos" o "despreciados" son algunas de las connotaciones peyorativas que reciben esas especies de propagación vegetativa. Por la importancia como materia prima en programas de mejoramiento, por la urgencia de evitar la erosión genética, estudios actuales con énfasis en marcadores moleculares trajeron, especialmente en los últimos años, informaciones importantes en relación con la diversidad genética mantenida por agricultores tradicionales y cómo esta diversidad se encuentra estructurada. Por la importancia que posee en Brasil, la caracterización y el análisis genético de la yuca, ñame y papa dulce (camote) harán el cuerpo central de esta revisión.

Escola Superior de Agricultura "Luiz de Queiroz", Departamento de Genética, Universidade de São Paulo, Brasil. mvbms@esalq.usp.br 


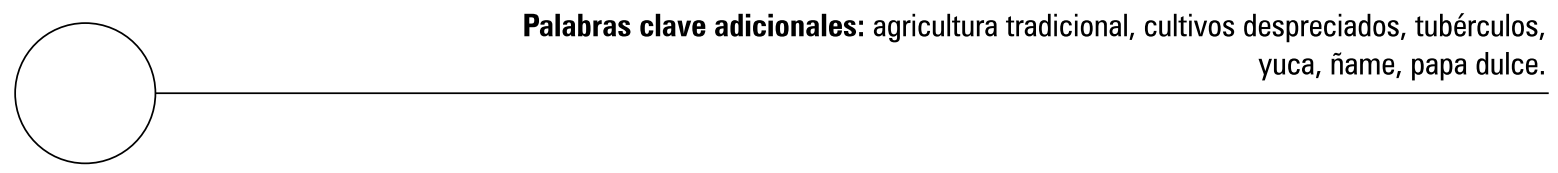

\section{ABSTRACT}

The traditional agriculture in Brazil basically is maintained for the needs of existence of local communities, without the use of modern technologies and employing the family labour. A wide scale of species is used by the farmers who support high diversity of varieties of each species, within or outside of the small farms, presenting also an intense system of exchanging of these genotypes among family members and neighbours. Of this high diversity of cultures, stand out tuberous root crops that are a part of the diet of the majority of Brazilians, being yucca, yam and sweet potato (camote) the most cultivated. In spite of all cultural and food importance, these tuberous crops demonstrate a "negligence" by the part of investigation groups, extensionists and society in general, taking into account that the investigations associated with these are scarce, if we compare these crops with the ones of short cycle culture, where predominate the strength of the agricultural business and governmental support. "Orphan" or "despised" cultures are some of the poor connotations that receive these species in part of vegetative propagation. Due to the importance of raw material in improvement programs, for the urgency of avoiding the genetic erosion, current studies with emphasis on molecular markers brought, especially in the last years, important information in relation to the genetic diversity supported by traditional farmers and the structure of this diversity. For the importance that these possess in Brazil, the characterization and genetic analysis of the yucca, yam and sweet potato (camote) will occupy the central volume of this review.

Additional keywords: traditional agriculture, underused crops, tubers, yucca, yam, sweet potato.
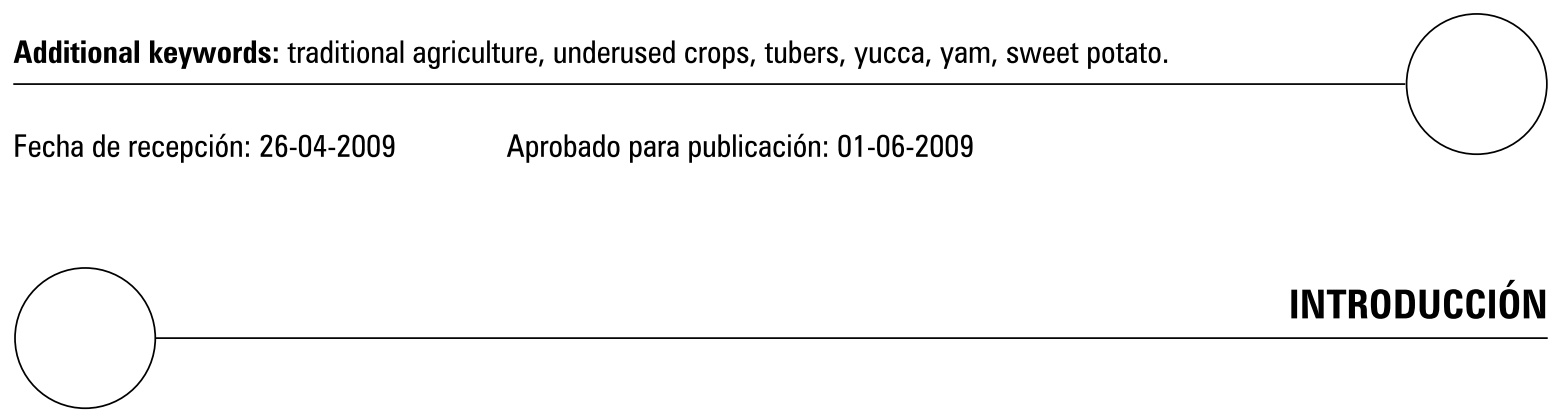

La chacra, unidad básica evolutiva de la agricultura tradicional, donde se desarrolla la conservación in situ (on farm) de las etnovariedades, o también denominadas variedades locales, y también donde existe ampliación de la diversidad genética, la cual beneficia a los agricultores locales (Martins, 2001), es un patrimonio agroecológico que se ha perdido a lo largo de los años. La migración de los pueblos a las grandes ciudades, la erosión de los suelos, el aumento del turismo, el uso abusivo del fuego para generar pastizales y el monocultivo, sobre todo de la soya, y la prohibición de la formación de claros en áreas de preservación ambiental ha llevado a muchas chacras al exterminio (Peroni y Hanazaki, 2002; Klink y
Machado, 2005; Jackson et al., 2007). Uno de los muchos beneficios que la agricultura tradicional ha proveído a la humanidad es la conservación in situ de germoplasma de muchas especies de importancia económica, manteniendo y amplificando en el tiempo la variabilidad genética (Martins, 1994; Peroni y Martins, 2000; Sambatti et al., 2001).

Entre las dos principales estrategias de conservación de germoplasma (in situ y ex situ), la menos exigente económicamente es la primera. Además de ser más barata, la conservación in situ, viabilizada por las chacras de agricultores tradicionales, es de fundamental importancia no sólo 
desde el punto de vista genético, sino también para la seguridad alimentaria y preservación del patrimonio cultural de muchas comunidades. Así, la conservación de los recursos genéticos in situ llevada cabo por los agricultores tradicionales tiene un papel fundamental, tomando en cuenta la estrechez de la base genética de las especies cultivadas que se viene manifestando en los últimos años, consecuencia del modelo de agricultura de la Revolución Verde y las limitaciones de la conservación ex situ, extremadamente onerosa $y$ de difícil mantenimiento (Clement et al., 1982; Amorozo, 2000, 2008).

La conservación in situ (en la chacra) se refiere a la selección y transformación genética continuas, en vez de preservación estática. Los cultivos así seleccionados se mantienen adaptados a las condiciones del ambiente local, de manejo y a todos los problemas bióticos del cultivo (plagas, enfermedades y hierbas dañinas). La conservación in situ requiere que las unidades de producción agrícola y los productores sean los depositarios tanto de la información genética como del conocimiento cultural de cómo los cultivos son cuidados y manejados. Por tanto, el principio de la conservación in situ propone que cada unidad de producción agrícola tenga su propio programa de mejoramiento y preservación. En realidad, los productores deben ser capaces de seleccionar y preservar sus propias etnovariedades adaptadas localmente, donde eso fuese posible. Como las características de una región establecen criterios importantes de selección, puede haber cierta centralización de programas de selección para una determinada área, definida ecológica y geográficamente, toda vez que el intercambio constante entre material genético de cultivos agrícolas ocurra entre los productores de aquella región. Esas unidades agrícolas son en gran parte áreas pequeñas y medianas donde es practicada de forma plena o con algunas adaptaciones modernas la agricultura tradicional de tipo tumba y quema (Altieri y Montecinos, 1993).

La agricultura de tipo tumba y quema está caracterizada generalmente por el uso de un ciclo de corte de la vegetación en estado de sucesión secundaria, seguido por la quema de esta vegetación cuando seca, plantío, cultivo, abandono del área después de la cosecha y el retorno al área después de algunos años (Éden, 1988). Segundo Diegues y Arruda (2001), varias culturas tradicionales existen en el Brasil y se debe a ellas que tenemos una gran amplitud de cultivos. Varias comunidades rurales encontradas en el país son resultado de un intenso mestizaje entre pueblos blancos, negros e indios (Arruda, 1999).

La introducción de varias especies y variedades de hortalizas, además de diversificar la alimentación de los primeros colonizadores, sirvió de material básico para el mejoramiento genético, en la búsqueda de una mejor adaptación de estas especies a las diferentes condiciones edafoclimáticas encontradas en el Brasil. Otras introducciones se volvieron indispensables a la culinaria regional en algunas regiones brasileras. Otra contribución es indirecta y poco honrosa para los descubridores portugueses, pero ocurrió de hecho y hoy resultó en el enriquecimiento aún mayor del mestizaje del pueblo brasilero, fue el flujo de ñames traídos por los esclavos africanos (Madeira et al., 2008). La yuca y la papa dulce (camote), cultivadas por pueblos indígenas de América del Sur, siempre fueron la base de sus dietas alimenticias y a partir de varios sistemas de cultivo tradicional han sido creadas variedades regionales a partir del conocimiento local, muchas veces centenario y poco comprendido (Brush, 2005). Fue a través de las rutas migratorias de pueblos indígenas y de colonizadores europeos que muchas tuberosas fueron incorporadas y ampliamente cultivadas hasta hoy. Actualmente, la diversidad genética de ciertas especies de hortalizas es mantenida por esos agricultores locales de forma que satisfacen sus necesidades, de acuerdo con el contexto social en el que están incluidos (Bellon, 1996; Veasey et al., 2007).

En Brasil, al igual que en otros países en desarrollo, especies como la yuca (Manihot esculenta), los ñames (Dioscorea spp.) y la papa dulce (Ipo- 
mea batatas), tienen una profunda importancia en la agricultura familiar, siendo utilizadas básicamente como fuente de carbohidrato, sobre todo en la región donde residen comunidades de bajo poder adquisitivo. Sus características van más allá de las altas cualidades nutritivas; esas especies también presentan importantes propiedades medicinales, muchas de ellas desconocidas entre nosotros. En Brasil, en algunos estados como Paraíba y Pernambuco, el ñame es un importante recurso alimenticio, difundido por todos los municipios. Ya la papa dulce o camote gana destaque en regiones del sur y sureste, con alguna importancia en el noreste (Silva et al., 2002). La yuca, en cambio, está difundida por todo el país, con una cadena productiva sui generis. Sin embargo, el gran porcentaje del material cultivado en territorio brasilero para ambas especies es de origen tradicional, con una gran diversidad de variedades. Lo que se encuentra en el mercado, en muchos casos, son cultivos poco o nada mejorados, desmereciendo la aceptación del consumidor.

Son pocas las instituciones actualmente involucradas en investigaciones relacionadas con el cultivo de la yuca, ñames y papa dulce (camote), siendo urgente el incremento de estudios e investigaciones profundas, para que se comprendan los factores que interfieren en la evolución de esas especies y cómo la diversidad genética de éstas se encuentra distribuida actualmente. La literatura existente sobre esas "hortalizas subutilizadas" es escasa y se encuentra alejada de la realidad, obligando al investigador e interesado por estos cultivos a un intenso ejercicio de análisis de lo que es información correcta y errónea. Partiendo de la realidad, son raros los proyectos de investigación siendo realizados en el país, y con el propósito de rescate y valorización en el que se busque el mejoramiento y conservación de esas especies, el Departamento de Genética de la Escuela Superior de Agricultura "Luiz de Queiróz" en la Universidad de São Paulo (ESALQ/USP), en los últimos años, a partir de su germoplasma de raíces y tubérculos, ha buscado contribuir con nuevas informaciones sobre la caracterización morfológica y molecular de especies de propagación vegetativa.

Se postula que la conservación genética de variedades tradicionales es parte de un conjunto de políticas cruciales en el mantenimiento de la biodiversidad global, podremos afirmar que algunas variedades locales desaparecerán más temprano o más tarde, trayendo perjuicio a la población, sobre todo desde el punto de vista alimenticio. La urgencia de más estudios en especies como la yuca, los ñames y la papa dulce o camote se hace evidente por los siguientes factores: agricultores $y$ mejoradores que cultivan tuberosas enfrentan una serie de dificultades generadas por la falta de informaciones técnicas sobre el manejo de estos cultivos, de cara a la escasez de investigación en esta área. Acciones conjuntas entre instituciones académicas, de investigación y extensión son fundamentales para revertir este proceso, de modo que se pueda crear profesionales capacitados, generando y difundiendo tecnologías para el mejoramiento de estos mismos cultivos. La rápida erosión genética de esos materiales, sobre todo por el aumento de los monocultivos y áreas de pastizales, y consecuentemente el abandono de las tierras por pequeños agricultores, lleva a la necesidad de rescate y preservación del conocimiento tradicional y paralelamente a la orientación de programas de conservación de germoplasmas in situ (Jarvis et al., 2000; Altieri, 2002). Eso nos lleva a creer que la yuca, los ñames y la papa dulce, a semejanza de otros cultivos difundidos en chacras tradicionales, merecen una mayor atención del dominio público.

Muchos investigadores afirman que apenas las plantas de mayor dinámica fisiológica son las que prevalecerán a los cambios climáticos severos, como es el caso de esas hortalizas, a veces señaladas como "alternativas" o "no convencionales". Se sabe que la mayoría de los sistemas productivos de esas tuberosas despreciadas se encuentra en pequeñas propiedades y su manejo es técnicamente poco desarrollado. Áreas de mayor explotación también se encuentran desarticuladas de cara a los nuevos recursos, perdiendo 
en productividad tanto por la falta de acceso a los tubérculos semillas y tallos de buena calidad, como por el mal uso de los recursos, en los cuales se incluyen el suelo, el agua y la mano de obra no calificada. Se suman a estos factores la falta de apoyo de los órganos de gobierno para el incentivo de la pequeña y mediana explotación y la inexistencia de una cadena de exportación para responder a la exigencia de un mercado internacional competitivo y exigente. En relación con los programas de mejoramiento, un largo camino se ha de recorrer, ya que ellos están reducidos a pocas instituciones. La yuca ha recibido mayor atención por los mejoradores, con varias instituciones involucradas con programas de mejoramiento en Brasil, como la Empresa Brasileira de Pesquisa Agropecuaria (Embrapa), el Instituto Agronómico (IAC) y la Empresa de Pesquisa Agropecuaria y Extensión Rural de Santa Catarina (Epagri), entre otras (Fukuda, 1999). Si los abordajes de pre y poscosecha fuesen diferentes, los (pocos) problemas fitopatológicos en esos cultivos serían reducidos, tornándose más fácil las perspectivas de exportación en el Brasil. La micropropagación, así como otras herramientas que la biotecnología ofrece, puede beneficiar a esos cultivos (Fregene et al., 2002).

En este contexto, al hacer la pregunta "?por qué continúan siendo esos cultivos marginalizados en Brasil ż" parece ser fácil la respuesta; difícil sin duda será trazar directrices en el sentido de mejoramiento y conservación de esas especies en el extenso territorio brasilero.

\section{YUCA}

La yuca (Manihot esculenta Crantz), Euphorbiaceae, es una de las principales plantas de interés económico del planeta, que ejerce importante papel en la alimentación de millones de personas, principalmente en los países tropicales, siendo también enormemente utilizada en la industria para fabricación de diversos productos. Además de la importancia económica, la yuca posee enorme peso cultural, toda vez que compone la dieta y el folklore de poblaciones tradicionales en diversos países.

Brasil, según Allem (2002), abriga a la mayoría de las especies (cerca de 55, lo que representa $80 \%$ de la diversidad). México viene en seguida, con 17 especies, y $15 \%$ de la diversidad. Se estima en 7.000 el número de variedades encontradas en todo el mundo (Mühlen et al., 2000).

Las dos especies de Manihot de mayor importancia económica son la $M$. esculenta, utilizada para alimentación, con raíces tuberosas que poseen alta capacidad de acumulación de almidón, y $M$. glaziovii, utilizada en Africa para la producción de latex (Cury, 1993). En relación con M. esculenta, existen dos tipos de yuca, clasificadas de acuerdo con la concentración de glucósidos cianogénicos, las yucas bravas, denominadas por los agricultores apenas como yuca, o yuca brava, y las yucas dulces, conocidas por macaxeira (Valle et al., 2004). Las yucas bravas son las más apropiadas para la fabricación de harina, y las yucas dulces son utilizadas en incontables recetas culinarias, sin embargo, ambas tienen otras innumerables aplicaciones (Cereda, 2003).

Desde el punto de vista agronómico, biológico y experimental, la yuca es una especie muy interesante, pues consiguió, incluso después de un intenso proceso de domesticación, mantener la rusticidad, sin perder la capacidad de reproducirse sexualmente. Si consideramos el proceso de domesticación como continuo, la domesticación de la yuca continúa ocurriendo, como ocurre en las chacras de la agricultura tradicional autóctona de todo el mundo (Cury, 1998). La agricultura tradicional, realizada por poblaciones autóctonas de los países tropicales, es de suma importancia para la evolución de la especie.

Cury (1993) estableció principios básicos para el establecimiento del modelo de la dinámica evolutiva de la yuca (Martins, 2001). El modelo utiliza la yuca como unidad evolutiva y lleva 
en consideración el hecho de que la especie se propaga vegetativamente, sin dejar de poseer un sistema reproductivo sexual activo, capaz de generar nuevos recombinantes, que aparecen en las chacras a través de la germinación espontánea de semillas.

De modo general, las chacras de yuca presentan elevada diversidad intra e inter variedades, sea en las chacras, en una misma comunidad, o entre comunidades (Peroni y Martins, 2000; Pereira, 2008), y varios trabajos refuerzan la tesis de que la Amazonía es uno de los principales centros de origen y diversidad de la especie en el planeta $(\mathrm{Ol}-$ sen y Schaal, 1999; Léotard, 2003; Olsen, 2004). Las diversas etnovariedades se diferencian en múltiples aspectos de interés ecológico y agronómico, como adaptación a condiciones adversas.

Para elucidar cuestiones relativas al origen y filogenia, los marcadores moleculares han sido importantes para la caracterización de la diversidad genética de la yuca, que también puede ser medida por indicadores morfológicos y agronómicos. Sambatti et al. (2000) estudiaron la diversidad fenotípica de etnovariedades provenientes de cuatro chacras autóctonas situadas en el municipio de Ubatuba (litoral del Estado de São Paulo), por medio de patrones isoenzimáticos y morfológicos. Concluyeron que hubo diversidad fenotípica, y que la diversidad isoenzimática se concentra básicamente en las chacras, lo que sugiere un gran flujo genético entre los agricultores estudiados. La diversidad de caracteres morfológicos tuvo una distribución similar a la encontrada para caracteres isoenzimáticos. De acuerdo con los resultados obtenidos, se cree que los caracteres morfológicos son objeto de selección por los agricultores, los cuales los utilizan como forma de cuantificar sus cultivos. Sambatti et al. (2001), evaluando la percepción de los agricultores en relación con la identificación de cultivos, compararon los nombres dados por ellos a los individuos encontrados con sus fenotipos isoenzimáticos y concluyeron que ellos tienen un sistema poco específico y generalizado para el reconocimiento de las variedades o morfotipos y tienden a subestimar la diversidad genética de la yuca en sus chacras.

En el Valle de Ribeira, Peroni (1998) concluyó, evaluando caracteres morfológicos e isoenzimáticos, que cada variedad está contenida en genotipos distintos, sin embargo, con gran semejanza morfológica, componiendo una población heterogénea. Cada etnovariedad puede entonces ser considerada una familia de genotipos. En ese estudio, él testificó que los agricultores diferencian correctamente las etnovariedades a través de la morfología. Entre tanto, en relación con los resultados obtenidos por Sambatti et al. (2001), los agricultores también subestiman la variabilidad en sus chacras, ya que no diferencian genotipos diferentes que presentan gran semejanza morfológica de las etnovariedades, dando el mismo nombre a genotipos diferentes.

Faraldo et al. (2000) evaluaron 141 etnovariedades de varios locales de Brasil a través de la técnica de electroforesis de isoenzimas, estudiando 11 sistemas enzimáticos. Los resultados revelaron índices elevados de heterozigosidad, observándose una mayor concentración de variabilidad en las chacras de la misma región y mayor variabilidad en las regiones que entre regiones. La diversidad intra específica está directamente relacionada con la estructura sociocultural del local. El grupo colectado en las chacras amazónicas presenta mayor diversidad genética.

Colombo et al. (2000) estudiaron la estructuración genética de etnovariedades de yuca provenientes de diversos locales de las Américas, utilizando marcadores RAPD (Random Amplified Polymorphic DNA). Encontraron baja estructuración genética entre los materiales evaluados, lo que fue explicado por los autores a través de la práctica común de intercambio de tallo. Analizando los genotipos en función de los datos climáticos de las localidades de origen, encontraron una estructuración en función de la temperatura y de la precipitación de esos locales. 
Pereira (2008), evaluando la diversidad y estructura genética en chacras de Amazonas a partir de marcadores microsatélites, concluyó que las chacras son extremadamente complejas desde el punto de vista genético, presentando altos valores de riqueza alélica, polimorfismo y heterozigosidad, sublineando que la diversidad está estructurada básicamente en cada chacra y entre las variedades de las diferentes chacras, consecuencia del intercambio de etnovariedades entre agricultores. Siqueira et al. (2009a), evaluando etnovariedades de diferentes regiones de Brasil, también con marcadores microsatélites, obtuvieron conclusiones semejantes, realzando que la migración de poblaciones puede responder a la mayor o menor proximidad genética entre los diferentes agrupamientos obtenidos.

Estudios como esos tienen como objetivo contribuir con una mayor comprensión sobre cómo la diversidad de la yuca se encuentra distribuida en diferentes regiones geográficas. Sus resultados se enfocan en promover la conservación in situ/on farm de un importante recurso genético a través de los agricultores tradicionales.

\section{ÑAME}

Dioscorea es el mayor género de la familia Dioscoreaceae, con cerca de 600 especies (Pedralli et al., 2002). Aunque sea elevado el número de especies de Dioscorea, seis de esas especies son consideradas más importantes en la alimentación humana: $D$. cayenensis Lam., D. alata L., $D$. bulbifera L., D. esculenta (Lour.) Burkill, D. rotundata Poir. y D. trifida L. De estas, apenas D. trifida es originaria del continente americano, teniendo como probable centro de origen a América Central, aunque Brasil también es considerado centro de origen de esta especie. Relatos revelan que, cuando colonizadores llegaron a América, los indios se alimentaban de la raíz "cará", nombre que tiene origen en la lengua tupi-guaraní (Abramo, 1990). Durante la expansión marítima y colonización de nuevos territorios en el siglo
XVI, portugueses y españoles tuvieron un papel importante en la dispersión de las otras especies cultivadas de Dioscorea, originarias de Africa tropical (complejo $D$. cayenensis/D. rotundata y $D$. bulbifera), India Central (D. alata y D. esculenta) y región indo-malaya (D. bulbifera) (Montaldo, 1991; Monteiro y Peressin, 2002).

El ñame, planta poliploide y de propagación vegetativa, constituye la alimentación básica para más de 100 millones de personas en todo el mundo, sobre todo en los trópicos húmedos y sub-húmedos (Mignouna et al., 2003b). A pesar de ser producido por todo el continente africano, Nigeria es conocida como el mayor productor de ñame del mundo, siendo este país responsable por $76 \%$ de la producción mundial, lo que representa aproximadamente 18,3 millones de toneladas. Datos totales apuntan para una producción africana de 28,8 millones de toneladas (Ike y Inoni, 2006).

La producción brasilera, en 1999, fue estimada en 225.000 t en una área cultivada de 24.500 ha (Monteiro y Peressin, 2002). La mayor producción de ñame en Brasil ocurre en el Nordeste, especialmente en los estados de Paraíba, Pernambuco, Alagoas, Bahía, Piauí, seguidos de otros de menor importancia. Los agricultores cultivan básicamente la especie $D$. cayenensis, conocida vulgarmente como "roxo-da-costa", aunque sean utilizados también algunos clones de D. alata, como el "cará São Tomé" y el ñame "corneta". Vale resaltar que ocurre en menor escala el cultivo de ñame "mimoso" o "inhambu" (D. trifida) y de ñame "hígado" (D. bulbifera) (Carvalho y Carvalho, 1999).

Las especies de Dioscorea son también cultivadas por sus propiedades medicinales (sapogeninas esteroidales, utilizadas en la producción de cortisona y hormonas sintéticas), en el siguiente orden de importancia: D. bulbifera, D. cayenensis, D. dumetorum (Kunth) Pax, D. alata, D. trifida, D laxiflora Griseb. y D. microbotrya Griseb. (PedraIli et al., 2002). Entre muchas de las propiedades 
medicinales del ñame, se destaca la prevención de enfermedades como la malaria, fiebre amarilla y dengue. La medicina tradicional utiliza este rizoma para erupciones de la piel, creyéndose en la limpieza de impurezas.

Los tubérculos de Dioscorea son ricos en carbohidratos y pobres en lípidos. Presentan, además de eso, importantes propiedades nutricionales, siendo ricos en proteínas, vitaminas $\mathrm{C}, \mathrm{B}_{1}$ (tiamina) y $B_{2}, B_{5}$ (niacina), riboflavinas, provitamina $\mathrm{A}, \beta$-caroteno, provitamina $\mathrm{D}$, almidón, ácido ascórbico, $\mathrm{Ca}, \mathrm{Fe}$ y $\mathrm{P}$, fitoesteroides, entre otras (Seagri, 2002). Su almidón es parecido al del maíz en sabor, textura y color. La harina puede ser adicionada a la de trigo para la fabricación de panes o puede ser utilizada en diversos platos, dulces o salados (Abramo, 1990).

Los ñames tienen gran importancia en la agricultura tradicional en Brasil (Peroni y Hanazaki, 2002; Bressan, 2005), así como en muchas pequeñas comunidades rurales de otros países (CBDC, 2001), distribuyéndose por los trópicos húmedos y semihúmedos (Lebot et al., 2005). La especie con mayor representación en la literatura científica y ampliamente consumida en Brasil es la $D$. alata, especie que fue domesticada en varios países, con gran aceptación tanto en el mercado local como en grandes superficies comerciales. Algunos trabajos con la finalidad de preservación en $D$. alata han sido realizados in vitro con éxito, porque su conservación en bancos de germoplasma, además de volverse dispendiosa, tiene los inconvenientes de los riesgos de ataques patogénicos y de erosión genética (Rodríguez et al., 2003). A pesar de toda la importancia alimenticia y medicinal, aún es un cultivo despreciado y mal comprendido.

Martin y Rhodes (1977) relataron que la clasificación intraespecífica de $D$. alata es problemática y las relaciones genéticas entre cultivos son difíciles de explicar. Años después Lebot et al. (1998) usaron marcadores isoenzimáticos para estudios de la diversidad genética en 269 cultivares de $D$. alata originarios del sur del Pacífico, Asia, África, Caribe y América del Sur, concluyendo que muchos cultivos exhibían variaciones diversas, muy probablemente debido al proceso de selección humana. Según los autores, la existencia de una variación genética significativa se debe también a las recombinaciones genéticas verificadas en los patrones isoenzimáticos.

En Brasil, los marcadores isoenzimáticos fueron usados para el estudio de la diversidad genética entre etnovariedades de ñames del Valle de Ribeira (Bressan, 2005), constatándose alta variabilidad genética mantenida por los agricultores de esta región, siendo que esa variabilidad no se encuentra estructurada en el espacio. Ya los marcadores RAPD fueron utilizados para evaluar la variabilidad intraespecífica en accesos originarios de Jamaica (Asemota et al., 1996) y en otras especies de Dioscorea de Africa, Asia y Polinesia (Ramser et al., 1996), caracterizando accesos importantes de Dioscorea como entre las especies D. cayenensis/D. rotundata (Dansi et al., 2000). A partir de estos marcadores, Munõz (2003) consiguió determinar qué genotipos de $D$. alata, de la colección de germoplasma del IIAP (Instituto de Investigación Agropecuaria de Panamá), se separaban claramente de los genotipos de la colección de germoplasma del Catie (Centro Agronómico Tropical de Investigación y Enseñanza).

El marcador AFLP (Amplified Fragment Length Polymorphism) fue también utilizado para evaluar la diversidad genética en $D$. alata y su relación con otras nueve especies comestibles de Dioscorea (Malapa et al., 2005) y en estudios sobre la domesticación del género Dioscorea (Scarcelli et al., 2006). Egesi et al. (2006), utilizando marcadores AFLP, consiguieron demostrar que, a partir de 53 accesos de D. alata, en África occidental y central, cada grupo formado era una mezcla de los accesos de origen geográfico diferente, indicando que la geografía no tuvo un papel central en la diferenciación de la especie. 
Otro marcador que ha sido utilizado para especies de Dioscorea son los microsatélites (Hochu et al., 2006; Bousalem et al., 2006). Fundamentalmente, los microsatélites han sido utilizados para estudios de los patrones de segregación de esos marcadores y caracterización de accesos de varias especies del género Dioscorea (Terauchi y Konuma, 1994; Mignouna et al., 2003a; Mignouna et al., 2003b; Scarcelli et al., 2005).

Con el objetivo de caracterizar especies cultivadas del género Dioscorea de frecuentes en áreas de agricultura tradicional de Brasil, enfatizando traer subsidios para la conservación on farm, se pueden citar nuevos estudios con $D$. trifida (Borges et al., 2007, 2008), D. cayenensis (Recchia et al., 2007) y D. bulbifera (Kreyci et al., 2008). Siqueira et al. (2009b) evaluaron la diversidad genética de etnovariedades de ñame colectadas en chacras de agricultura tradicional y de variedades comerciales obtenidas en ferias y mercados por medio de marcadores microsatélites, verificando alto polimorfismo entre los locus y gran variabilidad genética para el conjunto de accesos de D. alata. Con todo, en la investigación no observaron separación entre las etnovariedades y las variedades comerciales.

\section{PAPA DULCE (CAMOTE)}

La papa dulce o camote (Ipomoea batatas (L.) Lam.) es una planta de clima tropical o subtropical, también cultivada en regiones templadas. Es de fácil cultivo, rústica, de amplia adaptación, alta tolerancia a la sequía y bajo costo de producción. Los riesgos ofrecidos por el cultivo son mínimos si se compara a otras hortalizas. Es cultivada en toda la faja tropical del globo terrestre, donde tiene usos múltiples, tanto en la alimentación humana como animal, o como materia prima para la industria. Es la séptima más importante fuente de alimento en todo el mundo, junto con el trigo, el arroz, el maíz, la papa, la cebada y la yuca (CIP, 2009).
Actualmente, la papa dulce puede ser considerada como un cultivo asiático, ya que gran parte de la producción mundial es producida y consumida en Asia. Los países en desarrollo producen y consumen más de $95 \%$ del total mundial. Los mayores productores son China, Indonesia, India, Japón y Filipinas, siendo China el productor del 90\% del total mundial (CIP, 2009).

En Brasil, ella es cultivada en todo el territorio. Los mayores productores son los estados de Rio Grande do Sul, Santa Catarina, Bahía, Paraná y Rio Grande do Norte. Brasil produjo cerca de 500 mil toneladas en 2005 (IBGE, 2007) y las estimaciones apuntaban que ese número no sería muy diferente en los años siguientes.

El valor nutritivo del camote es alto, comparable al de la papa. Las raíces son buena fuente de energía (azúcares y otros carbohidratos), calcio, hierro y otros minerales y vitaminas, principalmente vitamina $C$. Algunos cultivos son ricos en vitamina A, particularmente las de pulpa anaranjada (Steinbauer y Kushman, 1971; Edmond y Ammerman, 1971; Kay, 1973; Folquer, 1978). La composición química varía con el cultivo, condiciones climáticas, época y periodo de cosecha, duración y condiciones de almacenamiento y tratos culturales (Edmond y Ammerman, 1971; Miranda et al., 1984; Cereda et al., 1984; Fischer y Karnatz, 1992).

La papa dulce y las especies relacionadas con ella son poco estudiadas, comparativamente a otros cultivos, del punto de vista de la citogenética, taxonomía, morfología, fisiología, distribución geográfica, sistemas de incompatibilidad, cruzamientos compatibles y otros. Naturalmente, estos tópicos son diferentes en especies silvestres y tipos cultivados, e importantes sobre varios aspectos, inclusive para elucidar y comprender el origen genético y la evolución de la especie. La existencia de innumerables especies silvestres del género Ipomoea y la gran difusión y diversidad de tipos existentes en toda América tropical, supe- 
rior a la encontrada en Oceanía (Yen, 1976) hace suponer que la domesticación de la papa dulce o camote sucedió en el continente americano (Folquer, 1978; Austin, 1977; FAO, 1981).

Uno de los trabajos más importantes sobre la evolución de I. batatas fue dirigido por Nishiyama (1982). Este autor tuvo sus trabajos de investigación basados en la compatibilidad sexual, síntesis de poliploides, análisis citogenéticos, comparaciones de genomas y características morfológicas y tuvo como principal premisa que la papa dulce se originó de I. trifida hexaploide por diferenciación génica, especialmente para la formación de raíces tuberosas. Las informaciones generadas por Nishiyama (1982) y sus colegas trajeron, en la época, un gran debate y mucha controversia. Entre tanto, sin entrar en mérito de la cuestión del origen genético, todos los investigadores son unánimes en reconocer el gran valor de las investigaciones conducidas por los científicos japoneses, cuyo trabajo es extremadamente importante, no sólo para elucidar y comprender el origen genético y la evolución de la papa dulce, sino sobre todo por la posibilidad que se abre con la utilización de los híbridos hexaploides $(2 \mathrm{n}=90)$, sintetizados a partir de otras especies del género Ipomoea, en programas de mejoramiento genético.

Lo que está en discusión aún hoy es el local exacto del centro de origen y domesticación: si es en el noroeste de América del Sur (Perú, Colombia y Ecuador) o el sur de México o América Central (principalmente Guatemala). Austin (1988), basado en análisis numéricos de características morfológicas en papa dulce o camote en especies silvestres de Ipomoea, había postulado que la papa dulce se habría originado en la región entre la Península de Yucatán, en México, y el Río Orinoco, en Venezuela. Estudios más recientes, basados en marcadores moleculares, indican a América Central como el centro primario de diversidad y el más probable centro de origen de la especie (Zhang et al., 2000; Gichuki et al., 2003). Perú y Ecuador deberían ser considerados como el centro secundario de la diversidad de la papa dulce (Zhang et al., 2000).

Brasil debería también ser considerado como un centro secundario de diversidad (Austin, 1988), una vez que varias especies de Ipomoea, de la sección papas, se encuentran en este país. Concordando con esta hipótesis, altos niveles de diversidad morfológica y molecular fueron observados entre etnovariedades del Valle de Ribeira, Estado de São Paulo, Brasil (Veasey et al., 2007, 2008). Usando ocho marcadores microsatélites, Veasey et al. (2008) verificaron gran polimorfismo, siendo que la mayor variabilidad genética se encontraba en las chacras. Los autores concluyeron que el gran intercambio de material entre agricultores, así como otros factores antrópicos, fueron determinantes para esos resultados. Oliveira et al. (2002), Daros et al. (2002) y Fabri (2009) también observaron elevada diversidad morfológica, isoenzimática y molecular entre variedades de papa dulce originarias de diferentes regiones de Brasil.

Entre 1940 y finales de los años sesenta, algunas instituciones de investigación mantienen colecciones de germoplasma e iniciaron programas de mejoramiento de papa dulce en Brasil. Entre ellas, se destacan el Instituto Agronómico (IAC), el antiguo Instituto de Ecología y Experimentación Agrícola, después Ipeacs-Itaguaí/ RJ, la Escola de Agronomia de Cruz das Almas (en el Estado de Bahía), y la Escola de Agronomia de Viçosa, hoy UFV (Estado de Minas Gerais), entre otras. En abril de 1980, se inició en Embrapa-Centro Nacional de Pesquisas en Hortalizas (CNPH), en Brasilia, D.F. un programa de mejoramiento genético de papa dulce (Miranda, 1983). Hoy, algunas instituciones de investigación en Brasil mantienen colecciones de germoplasma de papa dulce, existiendo un banco activo de germoplasma (BAG), mantenido por la Embrapa-CNPH, en Brasilia, otro en Embrapa-CNPMF, en Cruz das Almas, uno en el IAC, en Campinas, y uno en ESALQ/USP. 


\section{NOTAS FINALES}

Grandes áreas de preservación del territorio brasilero son ocupadas por poblaciones indígenas o de comunidades rurales "tradicionales" - caiçaras, ribeirinhos, seringueiros, quilombolas, caipiraspara las cuales la conservación de fauna y flora es una garantía de su perennidad. El proceso de expansión de las fronteras agrícolas y extractivistas de la sociedad brasilera, a su vez, reproduce y se pauta por un modelo de ocupación del espacio y de uso de los recursos naturales, generador de degradación ambiental y de enormes costos sociales, en particular para las sociedades rurales (Arruda, 1999).

El mantenimiento de la diversidad genética de los agroecosistemas tradicionales contribuye para la adaptabilidad de las poblaciones humanas, ya que provee garantías de sobrevivencia para el agricultor. Al mismo tiempo, a pesar del importante papel de las comunidades rurales para la conservación de la biodiversidad, innumerables factores se han constituido en riesgos para ésta, tales como presiones del mercado, éxodo rural y la globalización, que mina a las culturas locales (incluso los hábitos alimenticios, basados en el consumo de una pequeña variedad de alimentos) en pro de una supremacía cultural reduccionista, importada de los países desarrollados.
Nos parece claro que, a partir de los datos presentados, además de generadora de diversidad, la agricultura tradicional es también mantenedora de esas etnovariedades de yuca, ñame y papa dulce o camote. En el momento en que la acción humana deja de ejercer influencia directa, esas variedades locales permanecen en la naturaleza por poco tiempo o desaparecen en la competición con otras plantas silvestres. Este es un aspecto que diferencia a las plantas domesticadascultivadas de las plantas silvestres o salvajes y demuestra la estrecha relación entre las plantas cultivadas y el ser humano.

Para rescatar las etnovariedades de yuca, ñame y papa dulce y ampliar su uso en la agricultura, se torna necesario pensar en estrategias construidas a partir de un diagnóstico de las causas que vienen provocando el abandono de esas variedades locales. Tal diagnóstico puede ser iniciado a través de una lectura del espacio rural, y en especial de la situación socio-económica y ambiental en la cual la agricultura familiar está inserta. La compleja y alta diversidad genética de las tuberosas "despreciadas" revisadas aquí, y continuamente estudiadas en ESALQ/USP, realzan per se la importancia de cómo se encuentra el patrimonio hortícola nacional. No obstante, nuevos estudios se hacen necesarios para que las políticas de conservación in situ y on farm se puedan concretizar.

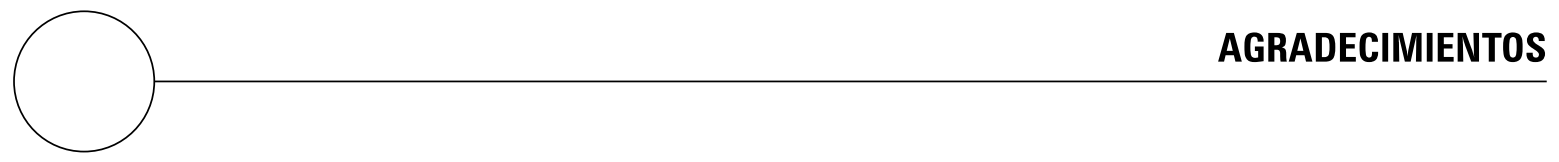

A Jorge Luis Ferrer Uribe, Eleonora Z. Blaco y Paulo Tavares de Melo por la ayuda en la revisión del manuscrito y valiosas sugerencias tanto en los aspectos de redacción como de contenido. 


\section{REFERENCIAS BIBLIOGRÁFICAS}

Allem, A.C. 2002. The origins and taxonomy of cassava. pp. 1-16. En: Hillocks, R.J.; J.M. Tresh y A.C. Bellotti (eds.). Cassava: biology, production and utilization. CABI Publishing, Oxon, UK.

Altieri, M. 2002. Agroecology: the science of natural resource management for poor farmers in marginal environments Agr. Ecosyst. Environ. 93, 1-24.

Altieri, M.A. y C. Montecinos. 1993. Conserving crop genetic resources in Latin America through farmers' participation. pp. 45-64. En: Potter, C.S.; J.I. Cohen y D. Janczewski (eds.). Perspectives on Biodiversity: Case Studies of Genetic Resource Conservation and Development. Washington D.C.

Abramo, M.A. 1990. Taioba, cará e inhame: o grande potencial inexplorado. Editora Ícone, São Paulo, Brasil.

Amorozo, M.C.M. 2000. Management and conservation of Manihot esculenta Crantz germplasm by traditional farmers in Santo Antonio do Leverger, Mato Grosso State, Brazil. Etnoecologica 4, 69-83.

Amorozo, M.C.M. 2008. Maintenance and management of agrobiodiversity in small-scale agriculture. Functional Ecosystems Communities 2, 11-20.

Arruda, R. 1999. "Populações tradicionais" e a proteção dos recursos naturais em unidades de conservação. Ambiente e Sociedade 2(5), 79-92.

Asemota, H.N.; J. Ramser; C. Lopéz-Peralta; K. Weising y G. Kahl. 1996. Genetic variation and cultivar identification of Jamaican yam germplasm by random amplified polymorphic DNA analysis. Euphytica $92,341-351$.

Austin, D.F. 1977. Hybrid polyploids in Ipomoea section batatas (Convolvulaceae). J. Heredity 68, 259-260.

Austin, D.F. 1988. The taxonomy, evolution and genetic diversity of sweetpotatoes and related wild species. pp. 27-60. En: Gregory, P. (ed.). Exploration, maintenance, and utilization of sweetpotato genetic resources. International Potato Center, Lima.

Bellon, M.R. 1996. The dynamics of crop infraspecific diversity: a conceptual framework at the farmer level. Econ. Bot. 50, 26-39.

Borges, A; G.H. Recchia y E.A. Veasey. 2007. Otimização de protocolo para amplificação de marcadores mi- crossatélites em Dioscorea trifida. En: Anais Simpósio Internacional De Iniciação Científica Da USP, Ribeirão Preto, Brasil.

Borges, A.; G.H. Recchia; L.C. Ming y E.A. Veasey. 2008. Role of traditional farmers in maintaining the genetic diversity assessed with microsatellites in $\mathrm{Di}$ oscorea trifida. En: Proc. Harlan II. An International Symposium. Biodiversity in Agriculture: Domestication, Evolution \& Sustainability, Davis, CA.

Bousalem, M.; G. Arnau; I. Hochu; R. Arnolin; V. Viader; S. Santoni y J. David. 2006. Microsatellite segregation analysis and cytogenetic evidence for tetrasomic inheritance in the American yam Dioscorea trifida and a new basic chromosome number in the Dioscoreae. Theor. Appl. Genet. 113, 439-451.

Bressan, E.A. 2005. Diversidade isoenzimática e morfológica de inhame (Dioscorea spp.) coletados em roças de agricultura tradicional do Vale do Ribeira - SP. Tese (Mestrado em Ecologia de Agroecossistemas) - Escola Superior de Agricultura "Luiz de Queiroz", Universidade de São Paulo, Piracicaba, Brasil.

Brush, S.B. 2005. Protecting traditional agricultural knowledge. Washington Univ. J. Law Policy 17, 59-109.

Carvalho, P.C.L. y R.L. Carvalho. 1999. Coleção de genótipos silvestres e cultivados de Dioscorea. En: Queiróz, M.A. de; C.O. Goedert y S.R.R. Ramos (eds.). Recursos Genéticos e Melhoramento de Plantas para o Nordeste brasileiro. Versão 1.0. Embrapa Semi-Árido, Petrolina-PE; Embrapa Recursos Genéticos e Biotecnologia, Brasília-DF, nov. 1999. http://www.cpatsa.embrapa.br; consulta: 22 de enero de 2007.

CBDC. 2001. Community Biodiversity Development and Conservation Programme - Bohol Project. Crop Genetic Resources Enhancement of Yam ( $D i-$ oscorea alata) in Four Towns in Bohol, Philippines. Technical Report No. 7. Southeast Asia Regional Institute for Community Education, Quezon City, Philippines.

Cereda, M.P. 2003. Tecnologia, usos e potencialidades de tuberosas amiláceas Latino Americanas. Fundação Cargill, Série Culturas de Tuberosas Amiláceas Latino Americanas. Vol 3. São Paulo, Brasil.

Cereda, M.P.; G. Wosiack y F.A.D. Conceição. 1984. Avaliação físico-química e reológica de vinte e seis cul- 
tivares de batata-doce (Ipomoea batatas (L.) Lam.). Hortic. Bras. 2(1), 6-12.

CIP - International Potato Center. 2009. Sweet potato production and consumption: major global impact. En: http://www.all-about-sweet-potatoes. com/production-consumption.html; consulta: 17 de junio de 2009 .

Clement, C.R.; W.E. Kerr y T. Ken-Hiri. 1982. Erosão cultural na Amazônia uma melhor adaptação às condições ecológicas da região. Ciência e Cultura 34, 1150-1153.

Colombo, C.; G. Second y A. Charrier. 2000. Diversity within of american cassava germplasm based on RAPD markers. Genet. Mol. Biol. 23, 189-199.

Cury, R. 1993. Dinâmica evolutiva e caracterização de germoplasma de mandioca (Manihot esculenta Crantz) na agricultura autóctone do sul do Estado de São Paulo. Piracicaba, 103 p. Dissertação (Mestrado) - Escola Superior de Agricultura "Luiz de Queiroz", Universidade de São Paulo, Brasil.

Cury, R. 1998. Distribuição da diversidade genética e correlações de caracteres em etnovariedades de mandioca (Manihot esculenta Crantz) provenientes da agricultura tradicional do Brasil. Piracicaba, 163 p. Tese (Doutrorado) - Escola Superior de Agricultura "Luiz de Queiroz", Universidade de São Paulo, Brasil.

Dansi, A.; H.D. Mignouna; J. Zoundjihékpon; A. Sangare; N. Ahoussou y R. Asiedu. 2000. Identification of some Benin Republic's Guinea yam (Dioscorea cayenensis/rotundata complex) cultivars using Randomly Amplified Polymorphic DNA. Genet. Resources Crop Evolution 47, 619-625.

Daros, M.; A.T. Amaral Júnior; T.N.S. Pereira; N.R. Leal; S.P. Freitas y T. Sediyama. 2002. Caracterização morfológica de acessos de batata-doce. Hortic. Bras. 20, 43-47.

Diegues, A.C.S. y R.S. Arruda. 2001. Saberes tradicionais e biodiversidade no Brasil. Ministério do Meio Ambiente, Brasília.

Eden, M.J. 1988. Crop diversity in tropical swidden cultivation: comparative data from Colombia and Papua New Guinea. Agr. Ecosyst. Environ. 20, 127-136.

Edmond, J.B. y G.R. Ammerman. 1971. Sweet Potato: Production, Processing, marketing. AVI Publishing, Westport, CT.
Egesi, C.N.; R. Asiedu; G. Ude; S. Ogunyemi y J.K. Egunjobi. 2006. AFLP marker diversity in water yam (Dioscorea alata L.) Plant Genet. Resources Characterization Utilization 4(3), 181-187.

Fabri, E.G. 2009. Diversidade genética entre acessos de batata-doce (Ipomoea batatas L. Lam.) avaliada através de marcadores microssatélites e descritores morfoagronômicos. Tese (Doutorado) - Escola Superior de Agricultura "Luiz de Queiroz", Universidade de São Paulo, Piracicaba, Brasil.

Faraldo, M.I.F; M.R. Silva; A. Ando y P.S. Martins. 2000. Variabilidade genética de etnovariedades de mandioca em regiões geográficas do Brasil. Scientia Agrícola 57, 499-505.

FAO - Food and Agriculture Organization of the United Nations. 1981. Genetic resources of sweet potato. IBPGR report, Roma.

Fischer, G. y A. Karnatz. 1992. Effect of soil temperature on growth and carbohydrates of two sweet potato (Ipomoea batatas) varieties from Papua-New Guinea. Plant Res. Dev. 35, 64-72.

Folquer, F. 1978. La batata (camote). Estudio de la planta y su producción comercial. Editorial Hemisfério Sur, Buenos Aires.

Fregene, M.; J. Tohme; W. Roca; P. Chavarriaga; R. Escobar y H. Ceballos. 2002. Biotecnología para la yuca. pp. 377-405. En: Ospina, B. y H. Ceballos (eds.). La Yuca en el Tercer Milenio. Sistemas Modernos de Producción, Procesamiento, Utilización y Comercialización. CIAT, CLAYUCA, Ministerio de Agricultura y Desarrollo y FENAVI, Cali, Colombia.

Fukuda, W.M.G. 1999. Melhoramento da mandioca. pp. 409-428. En: Borém, A. (ed.). Melhorameto de espécies cultivadas. Viçosa, UFV, Brasil.

Gichuki, S.T.; M. Berenyi; D. Zhang; M. Hermann; J. Schmidt; J. Glössl y K. Burg. 2003. Genetic diversity in sweetpotato [Ipomoea batatas (L.) Lam.] in relationship to geographic sources as assessed with RAPD markers. Genet. Resources Crop Evolution 50, 429-437. 429-437.

Hochu, I.; S. Santoni y M. Bousalem. 2006. Isolation, characterization and cross-species amplification of microsatellite DNA loci in the tropical American yam Dioscorea trifida. Mol. Ecol. Notes 6, 137-140.

IBGE, 2007. Brazilian Institute of Geography and Statistics. En: www.ibge.com.br 
Ike, P.C. y O.E. Inoni. 2006. Determinants of yam production and economic efficiency among smallholder farmers in southeastern Nigeria. J. Central Eur. Agr. 7, 337-342.

Jackson, L.E.; U. Pascual y T. Hodgkin. 2007. Utilizing and conserving agrobiodiversity in agricultural landscapes. Agr. Ecosyst. Environ. 121, 196-210.

Jarvis, D. I.; L. Myer; H. Klemick; L. Guarino; M. Smale; A.H.D. Brown; M. Sadiki; B. Sthapit y T. Hodgkin. 2000. A training guide for in situ conservation on-farm: version 1. International Plant Genetic Resources Institute, Rome, Italy.

Kay, D.E. 1973. Root crops. The Tropical Products Institute, London.

Klink, C.A. y R. Machado. 2005. Conservation of the Brazilian Cerrado. Conservation Biol. 19, 707-713.

Kreyci P.F.; O.M. Iamamoto; M.V.B.M. Siqueira y E.A. Veasey. 2008. Otimização de protocolo de amplificação de marcadores microssatélites em Dioscorea bulbifera para análises genéticas. En: Anais 16. Simpósio Internacional de Iniciação Científica da USP, Piracicaba.

Lebot, V.; R. Malapa; T. Molisale y J.L. Marchand. 2005. Physico-chemical characterisation of yam (Dioscorea alata L.) tubers from Vanuatu. Genet. Resources Crop Evolution 00, 1-10.

Lebot, V.; B. Trilles; J.L. Noyer y J. Modesto. 1998. Genetic relationships between Dioscorea alata L. cultivars. Genet. Resources Crop Evolution 45, 499-509.

Léotard, G. 2003. Phylogéographie et origine de la domestication du manioc (Manihot esculenta Crantz, Euphorbiaceae): les apports d'un échantillon élargi à l'écotone nord de l'Amazonie. DEA Biologie de l'Evolution et Ecologie, Université Montpellier II, Francia.

Madeira, N.R.; F.J.B. Reifschneider y L.B. Giordano. 2008. Contribuição portuguesa à produção e ao consumo de hortaliças no Brasil: uma revisão histórica. Hortic. Bras. 26, 428-432.

Malapa, R.; G. Arnau; J.L. Noyer y V. Lebot. 2005. Genetic diversity of the greater yam (Dioscorea alata L.) and relatedness to D. nummularia Lam. and D. transversa $\mathrm{Br}$. as revealed with AFLP markers. Genet. Res. Crop Evol. 52, 919-929.

Martin, F.W. y A. Jones. 1971. Flowering and fertility in six generations of open pollinated sweet potatoes. Amer. Soc. Hort. Sci. 96(4), 493-495.
Martin, F.W. y A. Jones. 1972. The species of Ipomoea closely related to the sweet potato. Econ. Bot. 26(3), 201-215

Martin, F.W. y A.M. Rhodes. 1977. Intra-specific classification of Dioscorea alata. Trop. Agr. 54, 1-13.

Martins, P.S. 1994. Biodiversity and agriculture: patterns of domestication of Brazilian native plants species. Anais da Academia Brasileira de Ciências 66, 219-224.

Martins, P.S. 2001. Dinâmica evolutiva em roças de caboclos amazônicos. pp. 369-384. En: Vieira, I.C.G.; J.M.C. Silva; D.C. Oren y M.A. D'incao (eds.). Diversidade Biológica e Cultural na Amazônia. Museu Paraense Emílio Goeldi, Belém, Brasil.

Mignouna, H.D.; M.M. Abang y S.A. Fagbemi. 2003a. A comparative assessment of molecular marker assays (AFLP, RAPD and SSR) for white yam (Dioscorea rotundata) germplasm characterization. Ann. Appl. Biol. 142, 269-276.

Mignouna, H.D; M.M. Abang y R. Asiedu. 2003b. Harnessing modern biotechnology for tropical tuber crop improvement: Yam (Dioscorea spp.) molecular breeding. Afr. J. Biotech. 2, 478-485.

Miranda, J.E.C. 1983. Programa de melhoramento da batata-doce (Ipomoea batatas (L.) Lam.) no CNPHortaliças. p. 135. En: Resumo Congresso Brasileiro de Olericultura, Rio de Janeiro, SOB, Brasil.

Miranda, J.E.C. y W.R. Maluf. 1984. Projeto de melhoramento genético da batata-doce. EMBRAPA-CNPH, Brasília (dados não publicados).

Montaldo, A. 1991. Cultivo de raíces y tubérculos tropicales. Instituto Interamericano de Ciências Agrícolas de la OEA, São José.

Monteiro, D.A. e V.A. Peressin. 2002. Cultura do inhame. pp. 511-518. En: Cereda, M.P. (coord.). Agricultura: tuberosas amiláceas Latino Americanas. Fundação Cargill, São Paulo, Brasil.

Mühlen, G.S.; P.S. Martins y A. Ando. 2000. Variabilidade genética de etnovariedades de mandioca, avaliada por marcadores de DNA. Scientia Agric. 57, 319-328.

Muñoz, Y.C.G. 2003. Caracterización morfológica y molecular de genotipos de Dioscorea alata y D. trifida del Instituto de Investigación Agropecuaria de Panamá, IDIAP y CATIE, Costa Rica. Tese (Mestrado em Educación para el Desarrollo y la Conservación). Centro Agronómico Tropical de Investigación y Enseñanza-CATIE, Costa Rica. 
Nishiyama, I. 1982. Autohexaploid evolution of the sweet potato. pp. 263-274. En: Villareal, R.L. e T.O. Grigs (eds.). Sweet potato: porceedings of the first international symposium. Shanhua, Tainan, Taiwan.

Oliveira, A.C.B.; M.A.N. Sediyama; T. Sediyama; F.L. Finger y C.D. Cruz. 2002. Variabilidade genética em batata-doce com base em marcadores isoenzimáticos. Hortic. Bras. 20, 576-582.

Olsen, K.M. 2004. SNPs, SSRs and inferences on cassava's origin. Plant Mol. Biol. 56, 517-526.

Olsen, K.M. y B.A. Schaal. 1999. Evidence on the origin of cassava: phylogeography of Manihot esculenta. Proceedings of the National Academy of Sciences 96, 5586-5591.

Pedralli, G.; C.A.S. Carmo; C. Marney y M. Puiatti. 2002. Uso de nomes populares para as espécies de Araceae e Dioscoreaceae no Brasil. Hortic. Bras. 20, 530-532.

Pereira, K.J.C. 2008. Agricultura tradicional e manejo da agrobiodiversidade na Amazônia Central: um estudo de caso nos roçados de mandioca nas Reservas de Desenvolvimento Sustentável Amanã e Mamirauá, Amazonas, Brasil. Tese (Doutorado) - Escola Superior de Agricultura "Luiz de Queiroz", Universidade de São Paulo, Piracicaba, Brasil.

Peroni, N. 1998. Taxonomia folk e diversidade intraespecífica de mandioca (Manihot esculenta Crantz) em roças de agricultura tradicional em áreas de mata atlântica no estado de São Paulo. Dissertação (Mestrado) - Escola Superior de Agricultura "Luíz de Queiróz", Universidade de São Paulo, Piracicaba, Brasil.

Peroni, N. y N. Hanazaki. 2002. Current and lost diversity of cultivated varieties, especially cassava, under swidden cultivation systems in the Brazilian Atlantic Forest. Agr. Ecosys. Environ. 92, 171-183.

Peroni, N. y P.S. Martins. 2000. Influência da dinâmica agrícola itinerante na geração de diversidade de etnovariedades cultivadas vegetativamente. Interciencia 25, 22-29.

Ramser, J.; C. López-Peralta; R. Wetzel; K. Weising y G. Kahl. 1996. Genomic variation and relationships in aerial yam (Dioscorea bulbifera L.) detected by random amplified polymorphic DNA. Genome 39, 17-25.
Recchia, G.H.; A. Borges y E.A. Veasey. 2007. Protocolo de amplificação de marcadores microssatélites em Dioscorea cayenensis Lam. para análises genéticas. In: Anais 15. Simpósio Internacional de Iniciação Científica da USP. Ribeirão, Brasil.

Rodríguez, A.; M. García; M. Borges; S. Meneses; N. Aguilera; J. Vázquez; Z. Infante y M. Fonseca. 2003. In vitro conservation of Dioscorea alata $\mathrm{L}$. germplasm by slow growth. Plant Genet. Resources Newsletter $133,8-12$.

Sambatti, J.B.M.; P.S. Martins y A. Ando. 2000. Distribuição da diversidade isoenzimática e morfológica da mandioca na agricultura autóctone de Ubatuba. Scientia Agric. 57, 75-80.

Sambatti, J.B.M.; P.S. Martins y A. Ando. 2001. Folk taxonomy and evolutionary dynamics of cassava: a case study in Ubatuba, Brazil. Econ. Bot. 55, 93-105.

Silva, J.B.C.; C.A. Lopes y J.S. Magalhães. 2002. Cultura da batata-doce. pp. 449-503. En: Cereda, M.P. (coord.). Agricultura: tuberosas amiláceas Latino Americanas, Vol. 2, Cap. 22. Fundação Cargill, São Paulo, Brasil.

Scarcelli, N.; O. Daïnou; C. Agbangla; S. Tostain y J.L. Pham. 2005. Segregation patterns of isozyme loci and microsatellite markers show the diploidy of African yam Dioscorea rotundata $(2 \mathrm{n}=40)$. Theor. Appl. Genet. 111, 226-232.

Scarcelli, N.; Tostain, S.; C. Mariac; C. Agbangla; O. Da; J. Berthaud y J. Pham. 2006. Genetic nature of yams (Dioscorea sp.) domesticated by farmers in Benin (West Africa). Genet. Resources Crop Evolution 53, 121-130.

Siqueira, M.V.B.M.; J.R.Q. Silva; E.A. Bressan; A. Borges; P. Kayo y E.A. Veasey. 2009a. Genetic characterization of cassava (Manihot esculenta) landraces in Brazil assessed with simple sequence repeats. Genet. Mol. Biol. 32, 104-110

Siqueira, M.V.B.M.; O.M. Iamamoto y E.A. Veasey. 2009b. Genetic characterization of landraces and commercial varieties of yam (Dioscorea alata) in Brazil with microsatellites markers. p. 51. En: Anais II Simpósio Brasileiro de Genética Molecular de Plantas, 1. Búzios, Brasil.

Steinbauer, C.E. y L.J. Kushman. 1971. Sweet-potato culture and diseases. Agriculture Handbook No. 388. USDA, Washington, D.C. 
Terauchi, R. y A. Konuma. 1994. Microsatellite polymorphism in Dioscorea tokoro, a wild yam species. Genome 37, 794-801.

Valle, T.L.; Carvalho, C.R.L.; M.T.B. Ramos; G.S. Mühlen y O.V. Villela. 2004. Conteúdo cianogênico em progênies de mandioca originadas do cruzamento de variedades mansas e bravas. Bragantia 63, 221-226.

Veasey, E.A.; Silva, J.R.Q.; M.S. Silva; A. Borges; E.A. Bressan y N. Peroni. 2007. Phenology and morphological diversity of sweet potato (Ipomoea batatas) landraces of the Vale do Ribeira. Scientia Agric. 64(4), 416-427.

Veasey, E. A.; A. Borges; M.S. Rosa; J.R. Queiroz; A.A. Bressan y N. Peroni. 2008. Genetic diversity in Bra- zilian sweetpotato (Ipomoea batatas (L.) Lam., Solanales, Convolvulaceae) landraces assessed with microsatellite markers. Genet. Mol. Biol. 31, 725-733.

Yen, D.E. 1976. Sweet potato - Ipomoea batatas (Convolvulaceae). pp. 42-45. En: Simmonos, N.W. (ed.). Evolution of crop plantas. Longman, London.

Wedderburn, M.M. 1967. A study of hybridization involving the sweet potato and related species. Euphytica $16,69-75$.

Zhang, D.P.; J. Cervantes; Z. Huamán; E. Carey y M. Ghislain. 2000. Assessing genetic diversity of sweet potato (Ipomoea batatas (L.) Lam.) cultivars from tropical America using AFLP. Genet. Resources Crop Evolution 47, 659-665. 